\title{
Synchronous Periampullary Tumors in a Patient With Pancreas Divisum and Neurofibromatosis Type 1
}

\author{
Cleandra Gregório 1,2, Clévia Rosset ${ }^{1}$, Laura da Silva Alves ${ }^{3}$, \\ Cristina Brinkmann Oliveira Netto ${ }^{4}$, Simone Marcia dos Santos Machado ${ }^{5}$, \\ Vivian Pierri Bersch ${ }^{6,7}$, Alessandro Bersch Osvaldt ${ }^{6,8,9}$ and Patricia Ashton-Prolla ${ }^{1,2,4 *}$
}

OPEN ACCESS

Edited by: Enrico Baruffini, University of Parma, Italy

Reviewed by: Irene Bottillo,

Ospedale San Camillo, Italy Rosa Nguyen, National Heart, Lung, and Blood Institute (NHLBI), United States Eric Pasmant

Université Paris Descartes, France

${ }^{*}$ Correspondence:

Patricia Ashton-Prolla pprolla@gmail.com

Specialty section: This article was submitted to Genetic Disorders,

a section of the journal

Frontiers in Genetics

Received: 05 December 2019 Accepted: 30 March 2020 Published: 28 April 2020

Citation:

Gregório $C$, Rosset $C$, Alves LdS, Netto CBO, Machado SMdS, Bersch VP, Osvaldt $A B$ and Ashton-Prolla P (2020) Synchronous Periampullary Tumors in a Patient With Pancreas Divisum and Neurofibromatosis Type 1. Front. Genet. 11:395 doi: 10.3389/fgene.2020.00395

\begin{abstract}
'Laboratório de Medicina Genômica, Centro de Pesquisa Experimental, Hospital de Clínicas de Porto Alegre, Porto Alegre, Brazil, ${ }^{2}$ Programa de Pós-graduação em Genética e Biologia Molecular, Universidade Federal do Rio Grande do Sul, Porto Alegre, Brazil, ${ }^{3}$ Faculdade de Medicina, Universidade Federal do Rio Grande do Sul, Porto Alegre, Brazil, ${ }^{4}$ Serviço de Genética Médica, Hospital de Clínicas de Porto Alegre, Porto Alegre, Brazil, ${ }^{5}$ Serviço de Patologia, Hospital de Clínicas de Porto Alegre, Porto Alegre, Brazil, ${ }^{6}$ Serviço de Cirurgia do Aparelho Digestivo, Grupo de Vias Biliares e Pâncreas, Hospital de Clínicas de Porto Alegre, Porto Alegre, Brazil, ${ }^{7}$ Grupo do Pâncreas, Serviço de Cirurgia do Aparelho Digestivo, Hospital Moinhos de Vento, Porto Alegre, Brazil, ${ }^{8}$ Programa de Pós-graduação em Medicina: Ciências Cirúrgicas, Universidade Federal do Rio Grande do Sul, Porto Alegre, Brazil, ${ }^{9}$ Faculdade de Medicina, Universidade Federal do Rio Grande do Sul, Porto Alegre, Brazil
\end{abstract}

Introduction: In this study, we describe for the first time a Neurofibromatosis type 1 patient with pancreas divisum, multiple periampullary tumors and germline pathogenic variants in NF1 and CFTR genes.

Case report: A 62-year-old female NF1 patient presented with weakness, choluria, nausea, and diffuse abdominal pain to an emergency room service. Magnetic resonance imaging revealed an abdominal mass involving the periampullary region and pancreas divisum. After surgical resection, three synchronous neoplasms were detected including two ampullary tumors (adenocarcinoma of the major ampulla and a neuroendocrine tumor of the minor ampulla) and a gastrointestinal stromal tumor (GIST). Germline multigene panel testing (MGPT) identified two pathogenic heterozygous germline variants: NF1 c.838del and CFTR c.1210-34TG[12]T[5].

Conclusion: This is the first report of a Neurofibromatosis type 1 patient with pancreas divisum and multiple periampullary tumors harboring pathogenic germline variants in NF1 and CFTR genes. The identification of two germline variants and a developmental anomaly in this patient may explain the unusual and more severe findings and underscores the importance of comprehensive molecular analyses in patients with complex phenotypes.

Keywords: synchronous neoplasms, GIST, periampullary tumors, neurofibromatosis type 1, pancreas divisum, CFTR pathogenic variant, NF1 pathogenic variant

\section{INTRODUCTION}

Pancreas divisum is a pancreatic duct developmental anomaly with an incidence of 4.5\% (Dimitriou et al., 2018). The anomaly is caused by absent or incomplete fusion of the ventral (main or Wirsung) and dorsal (marginal or Santorini) ducts (Kanth et al., 2014) and results in coexistence of two ampullary systems: the ventral duct drains the pancreatic head through the major ampulla, while the dorsal duct drains the pancreatic body and tail through the minor ampulla (Ferri et al., 2019). 
Ampullary neoplasms are rare, comprising $7 \%$ of all periampullary malignancies (Adsay et al., 2012), and association of theses tumors with pancreas divisum is considered an episodic event (Singh et al., 2003; Outtas et al., 2004; Kim et al., 2010). Their occurrence has been reported in families with hereditary cancer syndromes, such as Familial Adenomatous Polyposis (Pérez-Cuadrado-Robles et al., 2019) and Neurofibromatosis type 1 (NF1) (Tewari et al., 2014).

Neurofibromatosis type 1 (OMIM 162200) is one of the most common autosomal dominant disorders (incidence estimated at 1 in 2,500-3,000 live births) (Ferner et al., 2007). It is characterized by an increased risk of developing benign and malignant tumors, and a cumulative cancer risk of $20 \%$ in affected patients older than 50 years (Friedman and Birch, 1997; Upadhyaya, 2011). The NF1 gene product, neurofibromin, functions as a GTPase activating protein for RAS but has no Ras-GTPase activity, which regulates cell proliferation and differentiation (Cawthon et al., 1990; Boyd et al., 2009; Tewari et al., 2014). Germline heterozygous loss-of-function mutations in the NF1 gene lead to protein dysfunction and consequently, uncontrolled cell proliferation which has been associated with several of the NF1 clinical features. Loss of heterozygosity (LOH) has been reported as a necessary step for the development of malignancies in NF1 patients (Ruggieri and Packer, 2001). About one quarter of NF1 patients also have gastrointestinal involvement (Agaimy et al., 2012), with the occurrence of gastrointestinal stromal tumors (GISTs) and an increased incidence of neuroendocrine tumors (NET). The most frequent are somatostatin secreting duodenal NETs usually located in the periampullary region (Klein et al., 1989; Nunobe et al., 2003; Relles et al., 2010), followed by pheochromocytomas (Kalff et al., 1982) and pancreatic endocrine tumors (Thannberger et al., 2001; Fujisawa et al., 2002; Perren et al., 2006). A few NF1 patients with co-occurrence of GIST and NETs have been described in the literature (Tewari et al., 2014; Thavaraputta et al., 2019).

In this study, we describe and perform genetic analysis in a neurofibromatosis type 1 patient with pancreas divisum and multiple periampullary tumors. Although pancreas divisum is not a feature of NF1, the synchronous occurrence of multiple tumors in a NF1 background is extremely rare but more frequent than observed in the general population.

\section{CASE PRESENTATION}

A 62-year-old Caucasian female patient with NF1 presented to the emergency room with symptoms of weakness, nausea, vomiting, choluria, and diffuse abdominal pain. She reported a previous diagnosis of pheochromocytoma and breast cancer. Upon physical examination, the patient was jaundiced and had a palpable and painful gallbladder, and multiple neurofibromas in the abdomen and extremities. Initial laboratory findings showed unusually high levels of bilirubin (total bilirubin $9.9 \mathrm{mg} / \mathrm{dl}$ and direct bilirubin $8.4 \mathrm{mg} / \mathrm{dl}$ ), elevated serum C-reactive protein levels $(29.8 \mathrm{mg} / \mathrm{dl})$ and elevated liver enzymes.

Due to the high levels of bilirubin, the patient was submitted to an endoscopic retrograde cholangiopancreatography for placement of a biliary stent and during the procedure, an ulcerated expansive periampullary lesion was identified and biopsied. Pathology examination of the biopsy specimen revealed a moderately differentiated adenocarcinoma. During further investigation, an abdominal magnetic resonance imaging showed not only a small hypointense nodular mass (T2 sequence) in the periampullary region of the duodenum, but also pancreas divisum (Figures 1A,B).

The patient underwent a resection of the proximal jejunum, pylorus-preserving pancreaticoduodenectomy (PPPD) with routine reconstruction, and cholecystectomy. Macroscopic examination of the PPPD specimen revealed a $17.0 \times 2.0 \mathrm{~mm}$ ulcerated and infiltrative tumor located in the major ampulla (Figure 2A). In addition, a firm white lesion, $12.0 \mathrm{~mm}$ in diameter was detected in the duodenum (Figure 2B). Microscopic examination of the major ampulla revealed a poorly differentiated adenocarcinoma (pT2 pN1 R0) infiltrating the duodenal wall with focal necrosis (Figures 3A,B). Another lesion was identified in the minor ampulla corresponding to a well-differentiated NET (pT1 pN0 R0) $5.0 \mathrm{~mm}$ in diameter and without lymphovascular invasion (Figures 3C,D). The third synchronic tumor identified in the patient, a fusiform low-grade GIST, with a proliferative index of $2 \%$ (pT1 pN0 R0) was identified in the duodenal wall (Figures 3E,F). The patient was not eligible for chemotherapy or radiotherapy.
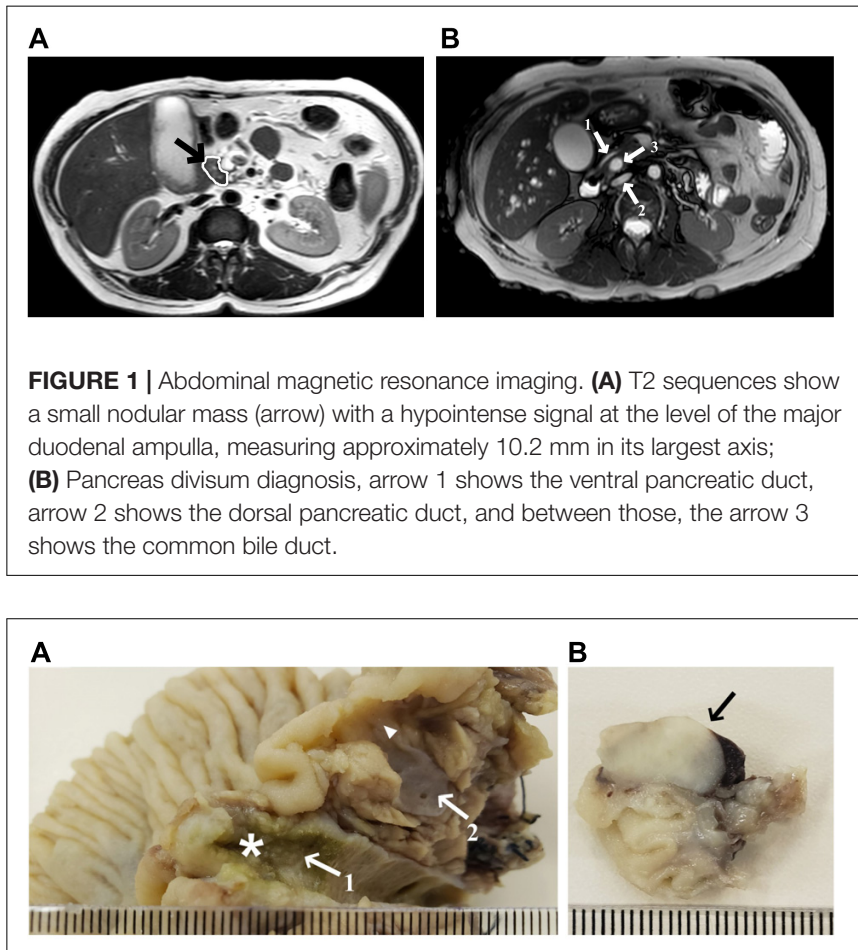

FIGURE 2 | Macroscopy specimens of pylorus-preserving pancreaticoduodenectomy. (A) Pancreas divisum, arrow 1 shows the dorsal duct with a vegetative lesion $(17.0 \times 2.0 \mathrm{~mm})$ extending to the major ampulla (star); arrow 2 shows the ventral duct and the arrowhead shows a poorly defined densification area near the minor ampulla (5.0 mm); (B) Duodenal wall mass $(12.0 \mathrm{~mm})$. 


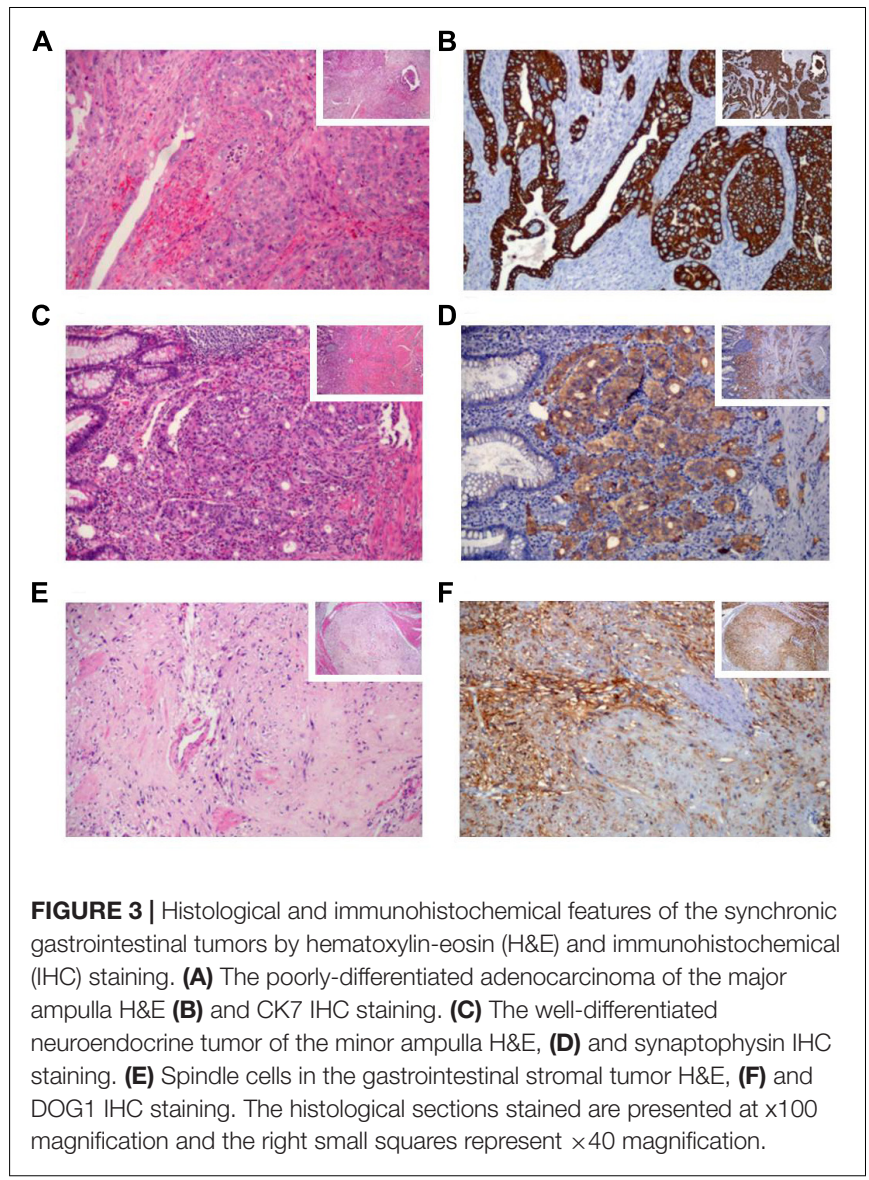

The patient reported a clinical diagnosis of NF1 since the age of 14 years and therefore, was referrd to the institutional genetic cancer risk assessment program. Physical examination identified multiple cutaneous neurofibromas and café-au-lait spots in the gluteal area, lower and upper limbs. Review of the past medical history was significant for diagnosis of viral hepatitis but she denied any previous history of pancreatitis. In addition, review of medical records and pathology reports confirmed previous occurrence of pheochromocytoma (at age 54 years), and breast cancer (first diagnosis at age 58 years and recurrence at age 61 years), all of which are features of the NF1 cancer predisposition syndrome. Review of the patient's family history revealed that her mother also had symptoms consistent with NF1 (café-au-lait spots and neurofibromas) although no formal clinical evaluation or molecular testing had been performed (Supplementary Figure S1A).

\section{MATERIALS AND METHODS}

\section{Multigene Panel Testing}

After signing a written informed consent for genetic analysis, a saliva sample was obtained with standard procedures using the Oragene DNA OG-500 self-collection kit (DNA Genotek, Ottawa, CA, United States) and the sample was submitted to germline genetic testing using a multigene panel test (MGPT) in a commercial laboratory (Invitae, San Francisco, CA, United States). The decision to investigate with a multigene panel was prompted by the presence of clinical features not consistent with NF1, such as pancreas divisum and by the diagnosis of five distinct primary tumors. Using Illumina technology, the presence of small variants, duplications and deletions in 29 genes (APC, ATM, BMPR1A, BRCA1, BRCA2, CDKN2A, EPCAM, MEN1, MLH1, MSH2, MSH6, NF1, PALB2, PMS2, SMAD4, STK11, TP53, TSC1, TSC2, VHL, CDK4, FANCC, PALLD, CASR, CFTR, CPA1, CTRC, PRSS1, and SPINK1) associated with hereditary pancreatic cancer and pancreatitis were assessed in the patient. All targeted regions were sequenced at a minimum of $50 \times$ depth and reads were aligned to a reference sequence (GRCh37) and with an average coverage depth of at least $350 \times$.

\section{Sanger Sequencing}

Sanger sequencing was performed to confirm the occurrence of the germline pathogenic variants found in MGPT testing. First, genomic DNA was extracted from patients' peripheral blood leucocytes using FlexiGene DNA kit (Qiagen, Hilden, Germany), according to the manufacturer's instructions. Specific primers for NF1 exon 8 and CFTR intron 9/exon 10 were designed using Primer Blast ${ }^{1}$ and used to amplify these regions by polymerase chain reaction (Supplementary Box S1). The reference sequences used were LRG_214 and LRG_663 for NF1 and $C F T R$, respectively. Sanger sequencing was performed on an ABI 3500 genetic analyzer (Thermo Fisher Scientific, CA, United States). Sequences were aligned to the reference sequences using CodonCode Aligner software implemented in MEGA 6.06.

\section{RESULTS}

Germline multigene panel testing revealed a novel heterozygous germline deletion in exon 8 of the NF1 gene, c.838del, p.(Ile280*) (NF1 chr17:29509630, NM_000267.3) which was reported as pathogenic since it predicts a premature stop codon, resulting in the absence of translation of all NF1 protein functional domains. This variant was not previously reported in the scientific literature, public variant databases (ClinVar and LOVD) or population databases (ExAC, Abraom, and 1000 genomes). Considering that more than 4000 different NF1 variants distributed along its 58 exons are reported to date in the ClinVar database, only a small portion are located in exon 8 (77 variants). Of these, only 16 are pathogenic. The significant allelic heterogeneity observed in NF1 renders genotype-phenotype correlations difficult, and no correlation between $N F 1$ variants in exon 8 and a specific phenotype have been established so far.

Additionally, one pathogenic germline variant was identified in heterozygosity in intron 9 of the CFTR gene, c.121034TG[12]T[5], (NM_000492.3). This sequence variant, also referred to as TG12-5T or T5TG12 in the literature, consists of $12 \mathrm{TG}$ and $5 \mathrm{~T}$ sequence repeats on the same chromosome and,

${ }^{1}$ http://www.ncbi.nlm.nih.gov/tools/primer-blast/ 
although it does not directly change the encoded amino acid sequence of the CFTR protein, it increases alternative splicing of exon 10 (referred to as exon 9 in some publications) from mRNA.

Sanger sequencing of peripheral blood DNA confirmed the germline deletion in NF1 exon 8 c.838del, p.(Ile280*) and the CFTR intron 9 variant, c.1210-34TG[12]T[5] (Supplementary Figure S1B,C).

\section{DISCUSSION}

It is well known that NF1 patients are predisposed to solid tumors, including those of the gastrointestinal tract (Agaimy et al., 2012), such as tumors arising in the ampulla of Vater and the duodenum. GIST, carcinoid tumors and other NETs are frequently reported in NF1 patients and they are considered part of the NF1 tumor spectrum, occurring in isolated manifestation or, in rare events, in synchronism with other tumors (Sørensen et al., 1986; Kim et al., 2014; Park et al., 2019). The co-occurrence of periampullary NET tumors and GIST has been proposed as highly suggestive or even pathognomonic of NF1 (Agaimy et al., 2012; Park et al., 2019; Poredska et al., 2019). LOH is a common event for the development of malignancies in NF1 patients, and has been considered by some as a prerequisite to cancer occurrence (Ruggieri and Packer, 2001). However, the development of specific tumors may require not only $\mathrm{LOH}$, but also additional genetic alterations (Patil and Chamberlain, 2012).

In this report, the patient was diagnosed with multiple tumors (GIST, NET of the minor ampulla, and adenocarcinoma of the major ampulla), of which GIST and NET are likely related to NF1, a diagnosis confirmed by germline genetic testing. Among the tumors identified, GIST represents the most common gastrointestinal tumor in NF1 patients (Poredska et al., 2019), accounting for 5 to $25 \%$ of all NF1 neoplasms (Zöller et al., 1997; Miettinen et al., 2006). The last update of duodenal and periampullary tumors in NF1 patients indicates that most $(60 \%)$ neoplasms arise in the duodenum, while $31 \%$ originate in the ampulla of Vater. Stratification by histology shows that GISTs correspond to $34 \%$ of duodenal tumors and the majority of ampullary tumors are neuroendocrine (40\%), while only $8 \%$ are adenocarcinomas (Relles et al., 2010). Although adenocarcinomas are not usually considered part of the NF1 tumor spectrum, their occurrence should be carefully evaluated in NF1 patients. In fact, adenocarcinomas associated with NF1 seem to be rare events and have been reported in only a few studies (Deschamps et al., 2010; Tewari et al., 2014). In addition to the diagnosis of NF1, an anatomical anomaly was identified in the patient: pancreas divisum. Pancreas divisum has been reported in some NF1 patients with periampullary tumors and in most of them it is an incidental finding, with no apparent relationship with the development of neoplasia or with the syndrome itself (Waisberg et al., 2006; Bhandari et al., 2015). In general, the majority of patients with pancreas divisum do not develop symptomatic disease and there is no evidence of a direct relationship between this developmental anomaly and cancer (Ferri et al., 2019).
In addition to the germline NF1 variant, a heterozygous germline pathogenic CFTR variant c.1210-34TG[12]T[5] in gene was identified. CFTR encodes the cystic fibrosis transmembrane conductance regulator, which is a membrane protein and chloride channel. Pathogenic variants in CFTR decrease ion channel function and cause extracellular mucus build-up; excessively thick and sticky mucus obstructs airways and pancreatic ducts, resulting in cystic fibrosis (Wang et al., 2014). Although heterozygous carriers of pathogenic CFTR variants do not develop cystic fibrosis, they may have an approximately 4-10-fold increased risk for pancreatitis and associated pancreatic injury due to elevated mucus levels, fibrosis, and cyst formation (Noone et al., 2001; Schneider et al., 2011; Steiner et al., 2011; Hegyi et al., 2016). On the other hand, acute and chronic pancreatitis and associated pancreatic injury are risk factors for the development of pancreatic cancer (Becker et al., 2014; Kirkegård et al., 2018). In this context, it has been reported that pancreas divisum acts as modulator of pancreatitis risk in carriers of additional genetic variants such as the one described here in the CFTR gene (Bertin et al., 2012; Gutta et al., 2019).

Thirteen to thirty seven percent of pancreatitis patients are heterozygous for CFTR mutations (Zoller et al., 2007). In a large population-based study that screened approximately 320.000 individuals for cystic fibrosis carrier status, the allelic frequency of the variant c.1210-34TG[12]T[5] in CFTR was 0.04 (Strom et al., 2004). The frequency of this variant in other databases is questionable, since metrics indicate poor data quality at this genomic position. Despite its high frequency in the general population, this intronic variant is a pathogenic variant, confirmed by functional studies, which show that it results in a non-functional CFTR protein through abnormal splicing (Delaney et al., 1993; Strong et al., 1993; Niksic et al., 1999; Groman et al., 2004; Bombieri et al., 2011). The polymorphic polypyrimidine $(\mathrm{Tn})$ is associated with a variable efficiency of exon 10 splicing, and among the three different major variants (5T, 7T, and 9T), T5 allele is associated with a high frequency of alternatively spliced CFTR mRNA with loss of exon 10 (Chu et al., 1993). In a mini-gene assay, the percentage of CFTR mRNA without exon 10 was $54 \%$ for TG[11]T[5], 72\% for TG[12]T[5] and $100 \%$ for TG[13]T[5] (Niksic et al., 1999). For these reasons, this TG[12]T[5] variant has been classified as pathogenic. The specific combination of pancreas divisum and pancreatitis was previously reported in a carrier of the same pathogenic variant (Dray et al., 2007; Montagnani et al., 2013). Moreover, several polymorphic changes in CFTR gene (even some with higher allele frequencies than TG[12]T[5]) have been associated with chronic pancreatitis (de Cid et al., 2010) and higher susceptibility of respiratory infections (Polgreen et al., 2018).

In this case report, we describe an unusual clinical presentation in an NF1 patient and emphasize the importance of a comprehensive analysis of potential risk factors in situations with atypical and/or complex phenotypes. In this particular case, the combination of factors associated with pancreatitis (pancreas divisum and the genetic change in CFTR) and the pathogenic variant in NF1 may have had a synergistic role to increase the risk for occurrence of multiple tumors. With the ever increasing availability of MGPT, identification of individuals 
with more than one pathogenic germline variant is expected to increase and the critical review of these variants in terms of their causal effect will be key to determine patient care and follow-up recommendations.

\section{CONCLUSION}

The current report, to our knowledge, is the first clinical description of an NF1 patient with pancreas divisum and multiple periampullary tumors. The occurrence of a pathogenic variant in NF1 and another pathogenic variant in CFTR in the same patient is also reported for the first time. The identification of two germline variants and a developmental anomaly in this patient may explain the unusual and more severe findings. However, whether the combination these factors increases risk for multiple tumors in an additive manner remains to be determined. The particular genetic status of this patient will require careful surveillance for lifetime cancer risk as well as appropriate genetic counseling for her relatives and underscores the importance of comprehensive genetic testing in patients with complex phenotypes.

\section{ETHICS STATEMENT}

Written informed consent was obtained from the patient for publication of this case report and accompanying images. The study was conducted in accordance with the Declaration of Helsinki and has been approved by the Scientific and Research Committee of Hospital de Clínicas de Porto Alegre (protocol number 13-0260).

\section{AUTHOR CONTRIBUTIONS}

CG conceived the work and conception design of the case report. CG and CR design of the draft the manuscript. CG, CR, and

\section{REFERENCES}

Adsay, V., Ohike, N., Tajiri, T., Kim, G. E., Krasinskas, A., Balci, S., et al. (2012). Ampullary region carcinomas: definition and site specific classification with delineation of four clinicopathologically and prognostically distinct subsets in an analysis of 249 cases. Am. J. Surg. Pathol. 36, 1592-1608. doi: 10.1097/PAS. 0b013e31826399d8

Agaimy, A., Vassos, N., and Croner, R. S. (2012). Gastrointestinal manifestations of neurofibromatosis type 1 (Recklinghausen's disease): clinicopathological spectrum with pathogenetic considerations. Int. J. Clin. Exp. Pathol. 5, 852-862.

Becker, A. E., Hernandez, Y. G., Frucht, H., and Lucas, A. L. (2014). Pancreatic ductal adenocarcinoma: risk factors, screening, and early detection. World J. Gastroenterol. 20, 11182-11198. doi: 10.3748/wjg.v20.i32.11182

Bertin, C., Pelletier, A.-L., Vullierme, M. P., Bienvenu, T., Rebours, V., Hentic, O., et al. (2012). Pancreas divisum is not a cause of pancreatitis by itself but acts as a partner of genetic mutations. Am. J. Gastroenterol. 107, 311-317. doi: 10.1038/ajg.2011.424

Bhandari, R., Riddiough, G., Lokan, J., Weinberg, L., Efthymiou, M., and Nikfarjam, M. (2015). Somatostatinoma of the minor papilla treated by local excision in a patient with neurofibromatosis type 1. JOP 16, 81-84. doi: 10.6092/ $1590-8577 / 2906$
$\mathrm{CN}$ were involved in patient recruitment. SM, LA, CN, VB, AO, and PA-P provided clinical data, were directly involved in the clinical follow-up and helped to draft the manuscript. LA and SM carried out and interpreted the imaging studies. All authors revised the manuscript critically, contributed with interpretation of the findings and gave final approval of the version to be published. PP supervised the work.

\section{FUNDING}

This work was funded in part by a Fundo de Incetivo a Pesquisa (FIPE), Hospital de Clinicas de Porto Alegre Grant (number 13-0260) and in part by Conselho Nacional de Desenvolvimento Cientifico e Tecnologico (CNPQ) Grants (numbers 424646/2016-1 and 307826/2017-1).

\section{ACKNOWLEDGMENTS}

Our sincere thanks to the patient for her participation.

\section{SUPPLEMENTARY MATERIAL}

The Supplementary Material for this article can be found online at: https://www.frontiersin.org/articles/10.3389/fgene. 2020.00395/full\#supplementary-material

FIGURE S1 | Pedigree of a family carrying variant NF1 c.838del and Sanger sequencing electropherogram from heterozygous germline variants. (A) Pedigree of the NF1 c.838del heterozygous germline variant. The arrow indicates the proband; current age is indicated in parenthesis. Sanger sequencing electropherogram from proband demonstrating (B) NF1 Exon 8 heterozygous germline variant (c.838del) and (C) CFTR Intron 9 heterozygous germline variant c. 1210-34TG[12]T[5].

BOX S1 | Primers and PCR conditions.

Bombieri, C., Claustres, M., De Boeck, K., Derichs, N., Dodge, J., Girodon, E., et al (2011). Recommendations for the classification of diseases as CFTR-related disorders. J. Cyst. Fibros. 10(Suppl. 2), S86-S102. doi: 10.1016/S1569-1993(11) 60014-3

Boyd, K. P., Korf, B. R., and Theos, A. (2009). Neurofibromatosis type 1. J. Am. Acad. Dermatol. 61, 15-16. doi: 10.1016/j.jaad.2008.12.051

Cawthon, R. M., Weiss, R., Xu, G. F., Viskochil, D., Culver, M., Stevens, J., et al. (1990). A major segment of the neurofibromatosis type 1 gene: cDNA sequence, genomic structure, and point mutations. Cell 62, 193-201. doi: 10.1016/00928674(90)90253-b

Chu, C. S., Trapnell, B. C., Curristin, S., Cutting, G. R., and Crystal, R. G. (1993). Genetic basis of variable exon 9 skipping in cystic fibrosis transmembrane conductance regulator mRNA. Nat. Genet. 3, 151-156. doi: 10.1038/ ng0293-151

de Cid, R., Ramos, M. D., Aparisi, L., García, C., Mora, J., Estivill, X., et al. (2010). Independent contribution of common CFTR variants to chronic pancreatitis. Pancreas. 39, 209-215. doi: 10.1097/MPA.0b013e3181bab679

Delaney, S. J., Rich, D. P., Thomson, S. A., Hargrave, M. R., Lovelock, P. K., Welsh, M. J., et al. (1993). Cystic fibrosis transmembrane conductance regulator splice variants are not conserved and fail to produce chloride channels. Nat. Genet. 4, 426-431. doi: 10.1038/ng0893-426 
Deschamps, L., Dokmak, S., Guedj, N., Ruszniewski, P., Sauvanet, A., and Couvelard, A. (2010). Mixed endocrine somatostatinoma of the ampulla of vater associated with a neurofibromatosis type 1: a case report and review of the literature. JOP 11, 64-68.

Dimitriou, I., Katsourakis, A., Nikolaidou, E., and Noussios, G. (2018). the main anatomical variations of the pancreatic duct system: review of the literature and its importance in surgical practice. J. Clin. Med. Res. 10, 370-375. doi: $10.14740 /$ jocmr3344w

Dray, X., Fajac, I., Bienvenu, T., Chryssostalis, A., Sogni, P., and Hubert, D. (2007). Association of pancreas divisum and recurrent acute pancreatitis with the IVS85T-12TG allele of the CFTR gene and CFTR dysfunction. Pancreas 35, 90-93. doi: 10.1097/MPA.0b013e318054771f

Ferner, R. E., Huson, S. M., Thomas, N., Moss, C., Willshaw, H., Evans, D. G., et al. (2007). Guidelines for the diagnosis and management of individuals with neurofibromatosis 1. J. Med. Genet. 44, 81-88. doi: 10.1136/jmg.2006.045906

Ferri, V., Vicente, E., Quijano, Y., Ielpo, B., Duran, H., Diaz, E., et al. (2019). Diagnosis and treatment of pancreas divisum: a literature review. Hepatobiliary Pancreat. Dis. Int 18, 332-336. doi: 10.1016/j.hbpd.2019.05.004

Friedman, J. M., and Birch, P. H. (1997). Type 1 neurofibromatosis: a descriptive analysis of the disorder in 1,728 patients. Am. J. Med. Genet. 70, 138-143. doi: 10.1002/(sici)1096-8628(19970516)70:2<138::aid-ajmg7>3.0.co;2-u

Fujisawa, T., Osuga, T., Maeda, M., Sakamoto, N., Maeda, T., Sakaguchi, K., et al. (2002). Malignant endocrine tumor of the pancreas associated with von Recklinghausen's disease. J. Gastroenterol. 37, 59-67. doi: 10.1007/s535-0028135-x

Groman, J. D., Hefferon, T. W., Casals, T., Bassas, L., Estivill, X., Des Georges, M., et al. (2004). Variation in a repeat sequence determines whether a common variant of the cystic fibrosis transmembrane conductance regulator gene is pathogenic or benign. Am. J. Hum. Genet. 74, 176-179. doi: 10.1086/381001

Gutta, A., Fogel, E., and Sherman, S. (2019). Identification and management of pancreas divisum. Expert Rev. Gastroenterol. Hepatol. 13, 1089-1105. doi: 10. 1080/17474124.2019.1685871

Hegyi, P., Wilschanski, M., Muallem, S., Lukacs, G. L., Sahin-Tóth, M., Uc, A., et al. (2016). CFTR: a new horizon in the pathomechanism and treatment of pancreatitis. Rev. Physiol. Biochem. Pharmacol. 170, 37-66. doi: 10.1007/112_ 20155002

Kalff, V., Shapiro, B., Lloyd, R., Sisson, J. C., Holland, K., Nakajo, M., et al. (1982). The spectrum of pheochromocytoma in hypertensive patients with neurofibromatosis. Arch. Intern. Med. 142, 2092-2098. doi: 10.1001/archinte. 142.12.2092

Kanth, R., Samji, N. S., Inaganti, A., Komanapalli, S. D., Rivera, R., Antillon, M. R., et al. (2014). Endotherapy in symptomatic pancreas divisum: a systematic review. Pancreatology 14, 244-250. doi: 10.1016/j.pan.2014.05.796

Kim, I. Y., Cho, M. Y., and Kim, Y. W. (2014). Synchronous multiple colonic adenocarcinomas arising in patient with neurofibromatosis type 1. Ann. Surg. Treat. Res. 87, 156-160. doi: 10.4174/astr.2014.87.3.156

Kim, Y. G., Kim, T. N., and Kim, K. O. (2010). Carcinoid tumor of the minor papilla in complete pancreas divisum presenting as recurrent abdominal pain. BMC Gastroenterol. 10:17. doi: 10.1186/1471-230X-10-17

Kirkegård, J., Cronin-Fenton, D., Heide-Jørgensen, U., and Mortensen, F. V. (2018). Acute pancreatitis and pancreatic cancer risk: a nationwide matchedcohort study in denmark. Gastroenterology 154, 1729-1736. doi: 10.1053/j. gastro.2018.02.011

Klein, A., Clemens, J., and Cameron, J. (1989). Periampullary neoplasms in von Recklinghausen's disease. Surgery 106, 815-819.

Miettinen, M., Fetsch, J. F., Sobin, L. H., and Lasota, J. (2006). Gastrointestinal stromal tumors in patients with neurofibromatosis 1: a clinicopathologic and molecular genetic study of 45 cases. Am. J. Surg. Pathol. 30, 90-96. doi: 10.1097/ 01.pas.0000176433.81079.bd

Montagnani, M., Cazzato, S., Mutignani, M., Cevenini, M., Guidetti, E., Zvi, I. B., et al. (2013). A patient with pancreas divisum, recurrent acute pancreatitis, and homozygosity for the cystic fibrosis transmembrane regulator-associated protein 5T allele. Clin. Gastroenterol. Hepatol. 11, 579-581. doi: 10.1016/j.cgh. 2013.02.012

Niksic, M., Romano, M., Buratti, E., Pagani, F., and Baralle, F. E. (1999). Functional analysis of cis-acting elements regulating the alternative splicing of human CFTR exon 9. Hum. Mol. Genet. 8, 2339-2349. doi: 10.1093/hmg/8.13.2339
Noone, P. G., Zhou, Z., Silverman, L. M., Jowell, P. S., Knowles, M. R., and Cohn, J. A. (2001). Cystic fibrosis gene mutations and pancreatitis risk: relation to epithelial ion transport and trypsin inhibitor gene mutations. Gastroenterology 121, 1310-1319. doi: 10.1053/gast.2001.29673

Nunobe, S., Fukushima, N., Yachida, S., Shimada, K., Kosuge, T., and Sakamoto, M. (2003). Clear cell endocrine tumor of the pancreas which is not associated with von Hippel-Lindau disease: report of a case. Surg. Today 33, 470-474. doi: 10.1007/s10595-002-2508-x

Outtas, O., Barthet, M., De Troyer, J., Franck, F., and Garcia, S. (2004). [Pancreatic panniculitis with intraductal carcinoid tumor of the pancreas divisum]. Ann. Dermatol. Venereol. 131, 466-469. doi: 10.1016/s0151-9638(04)93641-1

Park, E. K., Kim, H. J., Lee, Y. H., Koh, Y. S., Hur, Y. H., and Cho, C. K. (2019). Synchronous gastrointestinal stromal tumor and ampullary neuroendocrine tumor in association with neurofibromatosis type 1: a report of three cases. Korean J. Gastroenterol. 74, 227-231. doi: 10.4166/kjg.2019.74.4.227

Patil, S., and Chamberlain, R. S. (2012). Neoplasms associated with germline and somatic NF1 gene mutations. Oncologist 17, 101-116. doi: 10.1634/ theoncologist.2010-0181

Pérez-Cuadrado-Robles, E., Piessevaux, H., Moreels, T. G., Yeung, R., Aouattah, T., Komuta, M., et al. (2019). Combined excision and ablation of ampullary tumors with biliary or pancreatic intraductal extension is effective even in malignant neoplasms. U. Eur. Gastroenterol. J. 7, 369-376. doi: 10.1177/ 2050640618817215

Perren, A., Wiesli, P., Schmid, S., Montani, M., Schmitt, A., Schmid, C., et al. (2006). Pancreatic endocrine tumors are a rare manifestation of the neurofibromatosis type 1 phenotype: molecular analysis of a malignant insulinoma in a NF-1 patient. Am. J. Surg. Pathol. 30, 1047-1051. doi: 10.1097/ 00000478-200608000-00018

Polgreen, P. M., Brown, G. D., Hornick, D. B., Ahmad, F., London, B., Stoltz, D. A., et al. (2018). CFTR heterozygotes are at increased risk of respiratory infections: a population-based study. Open Forum Infect. Dis. 5:ofy219.

Poredska, K., Kunovsky, L., Prochazka, V., Dolina, J., Chovancova, M., Vlazny, J., et al. (2019). Triple malignancy (NET, GIST and pheochromocytoma) as a first manifestation of neurofibromatosis type-1 in an adult patient. Diagn. Pathol. 14:77. doi: 10.1186/s13000-019-0848-7

Relles, D., Baek, J., Witkiewicz, A., and Yeo, C. J. (2010). Periampullary and duodenal neoplasms in neurofibromatosis type 1: two cases and an updated 20-year review of the literature yielding 76 cases. J. Gastrointest. Surg. 14, 1052-1061. doi: 10.1007/s11605-009-1123-0

Ruggieri, M., and Packer, R. J. (2001). Why do benign astrocytomas become malignant in NF1? Neurology 56, 827-829. doi: 10.1212/wnl.56.7.827

Schneider, A., Larusch, J., Sun, X., Aloe, A., Lamb, J., Hawes, R., et al. (2011). Combined bicarbonate conductance-impairing variants in CFTR and SPINK1 variants are associated with chronic pancreatitis in patients without cystic fibrosis. Gastroenterology 140, 162-171. doi: 10.1053/j.gastro.2010.10.045

Singh, V. V., Bhutani, M. S., and Draganov, P. (2003). Carcinoid of the minor papilla in incomplete pancreas divisum presenting as acute relapsing pancreatitis. Pancreas 27, 96-97. doi: 10.1097/00006676-200307000-00013

Sørensen, S. A., Mulvihill, J. J., and Nielsen, A. (1986). Long-term follow-up of von Recklinghausen neurofibromatosis. Survival and malignant neoplasms. N. Engl. J. Med. 314, 1010-1015. doi: 10.1056/NEJM198604173141603

Steiner, B., Rosendahl, J., Witt, H., Teich, N., Keim, V., Schulz, H.-U., et al. (2011). Common CFTR haplotypes and susceptibility to chronic pancreatitis and congenital bilateral absence of the vas deferens. Hum. Mutat. 32, 912-920. doi: 10.1002/humu.21511

Strom, C. M., Crossley, B., Redman, J., Buller, A. Quan, F., Peng, M., et al. (2004). Cystic fibrosis screening: lessons learned from the first 320,000 patients. Genet med 2004; 6: 136-140. CFTR heterozygotes are at increased risk of respiratory infections: a population-based study. Open Forum Infect Dis. 5:ofy219. doi: 10.1093/ofid/ofy219

Strong, T. V., Wilkinson, D. J., Mansoura, M. K., Devor, D. C., Henze, K., Yang, Y., et al. (1993). Expression of an abundant alternatively spliced form of the cystic fibrosis transmembrane conductance regulator (CFTR) gene is not associated with a cAMP-activated chloride conductance. Hum. Mol. Genet. 2, 225-230. doi: $10.1093 / \mathrm{hmg} / 2.3 .225$

Tewari, N., Rollins, K., Gandhi, N., Kaye, P., and Lobo, D. N. (2014). Mixed periampullary adenocarcinoma and somatostatinoma with small bowel 
gastrointestinal stromal tumour in neurofibromatosis type 1. JOP 15, 600-603. doi: 10.6092/1590-8577/2844

Thannberger, P., Wilhelm, J. M., Derragui, A., Saraceni, O., and Kieffer, P. (2001). Von Recklinghausen's disease associated with pancreatic somatostatinoma. Presse Med. 30, 1741-1743.

Thavaraputta, S., Graham, S., Rivas Mejia, A. M., and Lado-Abeal, J. (2019). Duodenal somatostatinoma presenting as obstructive jaundice with the coexistence of a gastrointestinal stromal tumour in neurofibromatosis type 1: a case with review of the literature. BMJ Case Rep. 12:bcr-2018-226702. doi: 10.1136/bcr-2018-226702

Upadhyaya, M. (2011). Genetic basis of tumorigenesis in NF1 malignant peripheral nerve sheath tumors. Front. Biosci. 16:937-951. doi: 10.2741/3727

Waisberg, J., de Matos, L. L., Waisberg, D. R., dos Santos, H. V. B., Fernezlian, S. M., and Capelozzi, V. L. (2006). Carcinoid of the minor duodenal papilla associated with pancreas divisum: case report and review of the literature. Clinics 61, 365-368. doi: 10.1590/s1807-59322006000400017

Wang, Y., Wrennall, J. A., Cai, Z., Li, H., and Sheppard, D. N. (2014). Understanding how cystic fibrosis mutations disrupt CFTR function: from single molecules to animal models. Int. J. Biochem. Cell Biol. 52, 47-57. doi: 10.1016/j.biocel.2014.04.001
Zoller, H., Egg, M., Graziadei, I., Creus, M., Janecke, A. R., Löffler-Ragg, J., et al. (2007). CFTR gene mutations in pancreatitis: frequency and clinical manifestations in an Austrian patient cohort. Wien Klin Wochenschr. 119, 527-533. doi: 10.1007/s00508-007-0849-5

Zöller, M. E., Rembeck, B., Odén, A., Samuelsson, M., and Angervall, L. (1997). Malignant and benign tumors in patients with neurofibromatosis type 1 in a defined Swedish population. Cancer 79, 2125-2131. doi: 10.1002/(sici)10970142(19970601)79:11<2125::aid-cncr9>3.0.co;2-n

Conflict of Interest: The authors declare that the research was conducted in the absence of any commercial or financial relationships that could be construed as a potential conflict of interest.

Copyright (๑) 2020 Gregório, Rosset, Alves, Netto, Machado, Bersch, Osvaldt and Ashton-Prolla. This is an open-access article distributed under the terms of the Creative Commons Attribution License (CC BY). The use, distribution or reproduction in other forums is permitted, provided the original author(s) and the copyright owner(s) are credited and that the original publication in this journal is cited, in accordance with accepted academic practice. No use, distribution or reproduction is permitted which does not comply with these terms. 